\title{
Modifications to the resistive MHD spectrum due to changes in the equilibrium
}

\author{
T Van Doorsselaere and S Poedts \\ Centrum voor Plasma-Astrofysica, Celestijnenlaan 200B, B-3001 Leuven, Belgium \\ E-mail: tomvd@wis.kuleuven.be
}

Received 14 July 2006, in final form 11 January 2007

Published 13 February 2007

Online at stacks.iop.org/PPCF/49/261

\begin{abstract}
The normal-mode spectrum of the linearized resistive magnetohydrodynamics (MHD) operator is determined numerically for an inhomogeneous plasma slab. The spectrum consists of fast and slow magnetosonic and Alfvén sub-spectra. The obtained Alfvén sub-spectrum consists of discrete resistive Alfvén modes and an 'ideal quasi-mode' that originates from a surface mode due to a sharp density transition layer in the plasma. The quasi-mode interacts with the resistive Alfvén modes when the width of the transition layer is modified. Eventually, when the transition layer spreads over a substantial part of the plasma, the quasi-mode even loses its global or 'collective' behaviour and gradually transforms into an ordinary resistive Alfvén mode. In other words, it joins the resistive Alfvén sub-spectrum. Avoided crossings occur during the interactions of the ideal quasi-mode and the resistive spectrum.
\end{abstract}

\section{Introduction}

In an inhomogeneous plasma, in general, the local Alfvén frequency $\left(\omega_{A}=\vec{k} \cdot \vec{B} / \sqrt{\mu_{0} \rho}\right)$ changes with position. The macroscopic dynamics of such a plasma is described by the model of magnetohydrodynamics (MHD). In the linearized ideal MHD-equations that govern the small amplitude perturbations of this system, mobile regular singularities occur at the positions where the frequency matches the local Alfvén frequency and the slow magnetosonic cusp frequency. Those singularities give rise to two continuous sub-spectra of improper (nonsquare integrable) eigenmodes (Goedbloed and Poedts 2004). We focus here on the Alfvén sub-spectrum. Ideal 'quasi-modes' (or 'collective modes') are found as additional singularities of the Green's function obtained when solving the initial value problem using an inverse Laplace transform. These ideal quasi-modes represent global or collective modes (i.e. the plasma exhibits globally coherent motions) which are damped exponentially, even in ideal MHD. Clearly, such collective modes (with a complex frequency) cannot be eigenmodes of the ideal MHD differential operator, which is Hermitian hence can only have purely real 
(waves) or purely imaginary (instabilities) eigenvalues. This is the reason why these global modes are called ideal 'quasi-modes'.

As shown by Balet et al (1982), such quasi-modes correspond to natural, global oscillation modes of the plasma which can be easily excited. Ideal quasi-modes can originate from many different kinds of waves, e.g. surface waves, kink modes, fast and slow magnetosonic waves, discrete Alfvén waves, etc (Goedbloed and Poedts 2004). In the present paper, we focus on the quasi-modes originating from surface waves that live on a discontinuous plasma-plasma interface. When the discontinuous interface is replaced by a narrow transition layer, the ideal surface wave is weakly damped and thus becomes an ideal quasi-mode.

A real plasma, however, is seldom ideal. Non-ideal effects include finite electrical conductivity (resistivity), viscosity, radiative losses, thermal conduction, etc. In the present paper, we will only consider the effect of resistivity, i.e. a finite magnetic Reynolds number $R_{m}=\mu_{0} l_{0} v_{0} / \eta$, with $l_{0}$ and $v_{0}$ a typical length scale and velocity, respectively.

In a plasma with a finite electrical conductivity $(\eta \neq 0)$, the abovementioned singularities are removed from the equations and the ideal Alfvén continuum is abruptly replaced by a discrete spectrum of damped resistive modes which are located on welldefined curves in the complex frequency domain. The loci of these curves have been calculated numerically and analytically by Ryu and Grimm (1984) and Dewar and Davies (1984), respectively. For perturbations proportional to $\exp (\mathrm{i} \omega t)$, this Alfvén branch curve begins at a well-defined position on the imaginary axis in the complex $\omega$-plane, contains one or more bifurcation points (Riedel 1986) and evolves to specific points on the real axis, including the end points of the ideal continuum. These authors also showed that the position of these branches of the resistive spectrum in the complex frequency plane is independent of the resistivity $\eta$ in the limit $\eta \rightarrow 0$.

The position of the resistive modes themselves, however, does depend on the parameter $\eta$. When $\eta$ decreases, the resistive Alfvén modes move from the imaginary axis towards the real axis on the fixed branch curves. Thus, the damping of the resistive MHD-modes is proportional to $\eta$ (Kerner et al 1986), but these modes do not converge to the ideal continuum modes in the limit $\eta \rightarrow 0$, with some exceptions (such as the end points of the continuum).

Poedts and Kerner (1991), on the other hand, showed that the ideal quasi-modes correspond to proper eigenmodes of the resistive MHD operator. Moreover, these authors showed that this resistive MHD equivalent of the ideal quasi-mode does converge to its ideal weakly-damped counterpart in the limit of vanishing resistivity. In the present paper, the relation between this mode and the resistive Alfvénic sub-spectrum is investigated in more detail. In particular, the other limit is studied, i.e. the limit of strong damping. This limit can be taken in different ways. As a matter of fact, the damping of the quasi-mode depends not only on the plasma resistivity but also on the plasma inhomogeneity (the gradient of the local Alfvén frequency, i.e. the width of the transition layer) and the wave number. Our computations show that in the strong damping limit, in contrast to the weak damping limit result of Poedts and Kerner (1991), there is not such a clear one-to-one relationship between the resistive eigenmode and the ideal MHD quasi-mode, in the sense that any of the discrete resistive modes can transform into the quasi-mode depending on the background properties.

Our results are of particular importance for the research area of coronal loop seismology, because quasi-mode kink oscillations on a plasma cylinder are often used as a mathematical model for the observed oscillations in coronal loops. Such oscillations were first observed by Aschwanden et al (1999), Nakariakov et al (1999) and Schrijver et al (1999). Since then a lot of progress has been made by exploiting these oscillations to study the plasma in the immediate surroundings of the coronal loops ('coronal loop seismology'). Recently, for example, Nakariakov and Ofman (2001) determined the magnetic field in coronal 
loops, Aschwanden et al (2003) determined the density ratio between coronal loops and their surroundings and Andries et al (2005) estimated the density scale height in the corona.

Also recently, much effort has been put into better modelling such coronal loop oscillations. Van Doorsselaere et al (2004) extended the cylindrical loop models with a quasi-monolithic density to models with a more realistic smoothly varying density profile. They found that the analytical formula for the density profile may be extended to the regime where it is not strictly valid, within errors of $25 \%$. Furthermore the curvature was included by Van Doorsselaere et al (2004), and the effect of longitudinal density stratification was estimated by Andries et al (2005). The results in the latter paper were verified numerically for loops with a smooth density profile by Arregui et al (2005).

In this numerical paper, avoided crossings were observed in the eigenvalues when the equilibrium parameters were changed. The paper by Van Doorsselaere et al (2005), in preparation was referred to in this context, by which is meant the current article.

The current paper will study the abovementioned avoided crossings in more detail in simpler configurations.

\section{Equilibrium and equations}

We solved the fully resistive, linearized, undimensionalized MHD-equations in a plane geometry. To do this, we used the LEDA-code (Kerner et al 1985). The code calculates eigenvalues of the system and assumes all quantities to be proportional to exp $(\lambda t)$. The real part of $\lambda$ thus expresses the damping, while the imaginary part is related to the frequency of the calculated eigenmode.

In the numerical code, we consider a one-dimensional equilibrium, i.e. all quantities depend on the $x$-coordinate only. The other spatial directions $y$ and $z$ are Fourier-analysed and have wave numbers $k_{y}$ and $k_{z}$, respectively. In the equilibrium, we consider a homogeneous magnetic field pointing in the $z$-direction, $\vec{B}=B \vec{e}_{z}$.

Additionally, we assume that the gas pressure vanishes. Thus, we exclude the slow magnetosonic waves from the analysis. Analogously to the profile used by Ruderman and Roberts (2002), we consider a density profile, which is constant (but different) at the left and right edge of the plasma and connects these two regions continuously with a sine-profile:

$\rho= \begin{cases}\rho_{\mathrm{l}}, & \text { for } 0 \leqslant x<s-\frac{l}{2}, \\ \frac{\rho_{\mathrm{l}}}{2}\left[\left(1+\frac{1}{\zeta}\right)-\left(1-\frac{1}{\zeta}\right) \sin \frac{\pi}{l}(x-s)\right], & \text { for } s-\frac{l}{2} \leqslant x \leqslant s+\frac{l}{2}, \\ \rho_{\mathrm{r}}, & \text { for } s+\frac{l}{2}<x \leqslant 1 .\end{cases}$

Here, $\rho_{\mathrm{l}}$ and $\rho_{\mathrm{r}}$ are the (constant) densities, respectively, left and right of the interface, $\zeta=\rho_{\mathrm{l}} / \rho_{\mathrm{r}}, l$ is the thickness of the interface and $s$ is the mean position of the interface. When $l=0$, we have a discontinuous density jump at $x=s$. On the other hand, $l=1$ corresponds to a fully inhomogeneous plasma.

The use of the mentioned density profile allows for resonant absorption (at least, when $l \neq 0$ ). Thus, in the calculated spectra a quasi-mode will be present.

In what follows, we take $\zeta=9$ and $B=1$. We also take $s=0.5$ to minimize the influence of the walls at $x=0$ and $x=1$. As boundary conditions at these positions, we impose that the radial velocity and radial component of the magnetic field are zero. Moreover, the radial components of the current and the temperature perturbation are set to zero. 

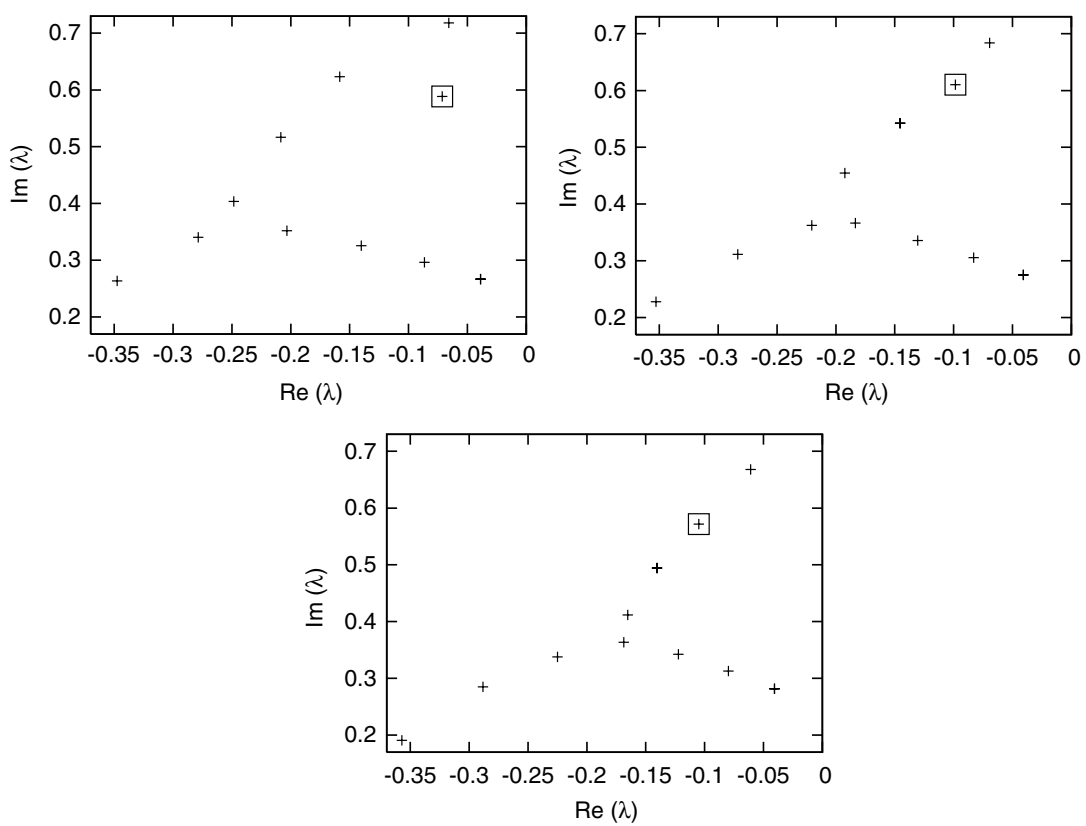

Figure 1. Snapshots of the resistive spectrum as $l$ increases. The horizontal axis is $\operatorname{Re}(\lambda)$, while the vertical axis is $\operatorname{Im}(\lambda)$. The quasi-mode is indicated with a $\square$. Figures shown for $l=0.63$ (upper left), $l=0.79$ (upper right) and $l=0.94$ (bottom). In each panel $\log \eta=-3.2, k_{z}=0.25$ and $k_{y}=1$.

\section{Modifications to the spectrum}

In this section, we study the evolution of the resistive spectrum when the thickness of the inhomogeneous layer $l$ increases. In each case, we keep $k_{y}$ and $k_{z}$ constant.

A known feature of quasi-modes is that when the thickness of the inhomogeneous layer increases, the quasi-modes become more heavily damped. This was shown by Ruderman and Roberts (2002) for small damping and was extended to large damping in a numerical study by Van Doorsselaere et al (2004). Thus, it could be expected that the frequency of the quasi-mode does not change very much, while the damping increases approximately linearly as $l$ increases. The expected result of widening the inhomogeneous layer would be that the quasi-mode frequency moves on a horizontal line to the left in the complex $\lambda$-plane.

Figure 1 shows a few snapshots of the behaviour of the resistive spectrum as $l$ increases, whereas figure 2 overplots all these snapshots in one frame. In figure 2, the actual trails of the frequencies in the complex plane as $l$ increases are shown.

As can be seen in the abovementioned figures, because of the change of the equilibrium, the outer resistive Alfvén modes together with the bifurcation point move inwards and the branches of the resistive Alfvén spectrum are pinched together. On the other hand, the quasi-mode moves initially to the left, which is the expected behaviour. However, when the quasi-mode gets too close to the branches of the resistive Alfvén spectrum, this behaviour is dramatically changed. The path of the quasi-mode frequency is bent and is now parallel to the paths of the resistive Alfvén modes. When $l$ has increased far enough, there is no obvious difference anymore between the original quasi-mode and the resistive point spectrum. It appears that the quasi-mode has been transformed into an ordinary discrete resistive mode. 


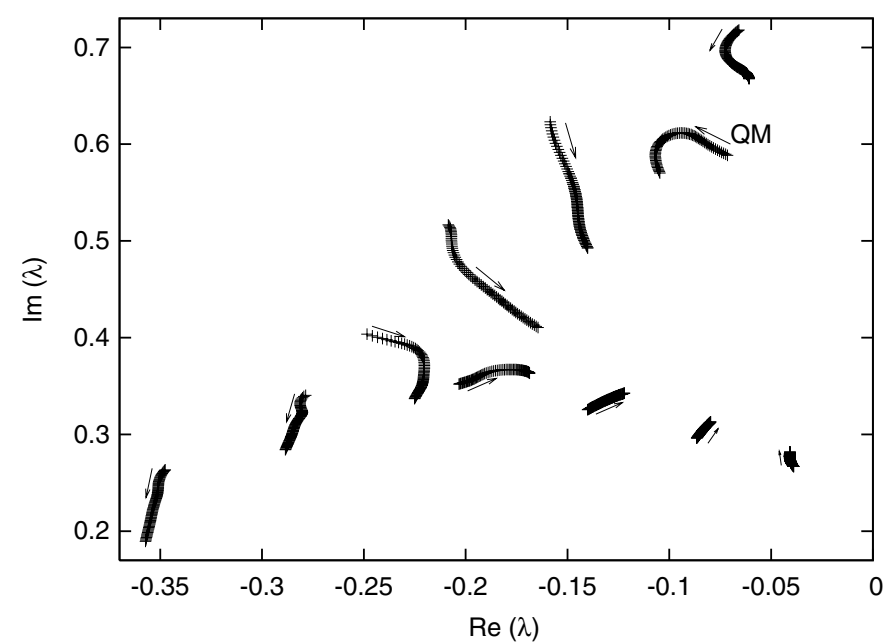

Figure 2. The resistive spectrum as $l$ increases. The horizontal axis is $\operatorname{Re}(\lambda)$, while the vertical axis is $\operatorname{Im}(\lambda)$. The figures are for $l$ from 0.63 increasing to $0.94 . \log \eta=-3.2, k_{z}=0.25$ and $k_{y}=1$. The arrows indicate the starting point of the frequencies (i.e. the spectrum for $l=0.63$ ) and their initial direction as $l$ increases. The path of the quasi-mode is indicated with QM.

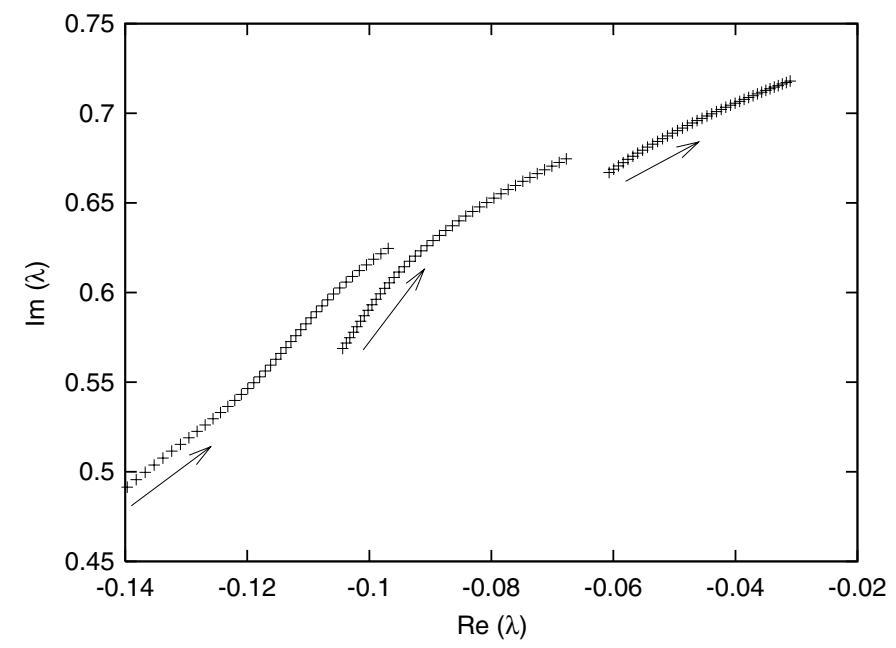

Figure 3. Part of the resistive spectrum as $\eta$ decreases. The horizontal axis is $\operatorname{Re}(\lambda)$, while the vertical axis is $\operatorname{Im}(\lambda)$. The figures are for $\log \eta$ from -3.2 to $-4.0 . \quad l=0.95, k_{z}=0.25$ and $k_{y}=1$. The arrows indicate the starting frequency for $\log \eta=-3.2$ and show the initial direction as $\eta$ decreases. The middle trail is the original quasi-mode.

This so far unobserved feature can also be confirmed by studying the behaviour of the spectrum when $\log \eta$ is being decreased and $l$ is large enough (see figure 3 ). In this figure, the original quasi-mode (middle) and its two neighbouring resistive Alfvén modes are followed as $\eta$ decreases. They all start at the lower left and move to the upper right (which is the expected behaviour for resistive Alfvén modes). It is clear that the behaviour of the original quasi-mode is similar to the behaviour of the discrete resistive modes. If the quasi-mode properties are still present, the value of the resistivity would not influence the complex quasi-mode frequency. 


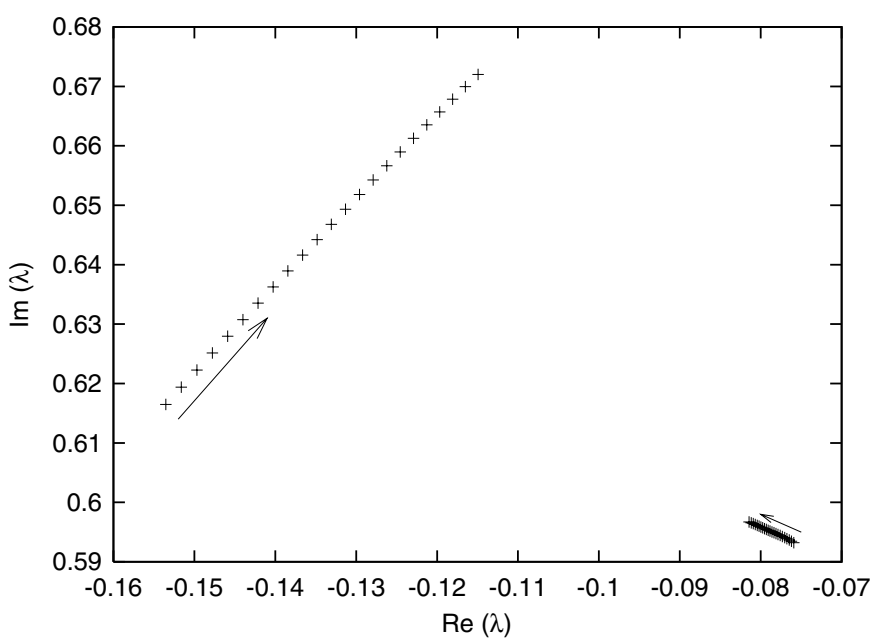

Figure 4. Part of the resistive spectrum as $\eta$ decreases. The horizontal axis is $\operatorname{Re}(\lambda)$, while the vertical axis is $\operatorname{Im}(\lambda)$. The figures are for $\log \eta$ from -3.22 to -3.44 . $l=0.65, k_{z}=0.25$ and $k_{y}=1$. The arrows indicate the starting frequency for $\log \eta=-3.22$ and show the initial direction as $\eta$ decreases. The trail on the left is Alfvénic, whereas the quasi-mode is found at the right.

This well-known feature is confirmed and illustrated in figure 4. In this figure, the resistive Alfvén mode moves from the lower left to the upper right. However, in agreement with Poedts and Kerner (1991), the quasi-mode is only slightly influenced by the resistivity.

We can conclude that the original quasi-mode must have interacted with the neighbouring Alfvén modes and must have somehow lost its quasi-mode properties. Note that the quasimode studied here originates from a surface mode. As the width of the inhomogeneous layer is increased, the 'surface' (limit $l \rightarrow 0$ ) completely disappears and thus the quasi-mode also disappears.

Another feature visible in figure 2 is that the resistive Alfvén modes, which are closest to the quasi-mode, do not follow the straight line, that is normally followed by the resistive modes when pinching the triangle by changing the equilibrium. It appears that they move aside to allow the quasi-mode to take up position between them. Before the interaction has started, the distance along the branches of the resistive Alfvén spectrum between subsequent modes is approximately equal. During the interaction, the neighbouring resistive Alfvén modes move aside to make sure that after the interaction, the distance along the branches between the modes is again equally distributed.

A possible explanation for the unexpected path of the quasi-mode (and the neighbouring resistive Alfvén modes) is an 'avoided crossing' phenomenon. When the original quasi-mode has a close encounter with a resistive mode, it passes its quasi-mode properties on to the resistive Alfvén mode and gets in exchange an Alfvénic character.

For some parameter values, convincing evidence for avoided crossings can be found in $(\operatorname{Re}(\lambda), l)$ - and $(\operatorname{Im}(\lambda), l)$-plots. In figure 5, a clear case of such an avoided crossing is displayed. In the top right panel of this figure, where the imaginary part of $\lambda$ is plotted versus $l$, the three Alfvén modes initially follow approximately parallel decreasing straight lines. On the other hand, the frequency of the original quasi-mode increases for $l<0.8$. However, when the original quasi-mode enters the neighbourhood of the top Alfvén mode, its path is bent and becomes parallel to the original Alfvénic paths $(l>0.85)$. After the exchange of wave properties, the path of the interacting resistive Alfvén mode is also bent 

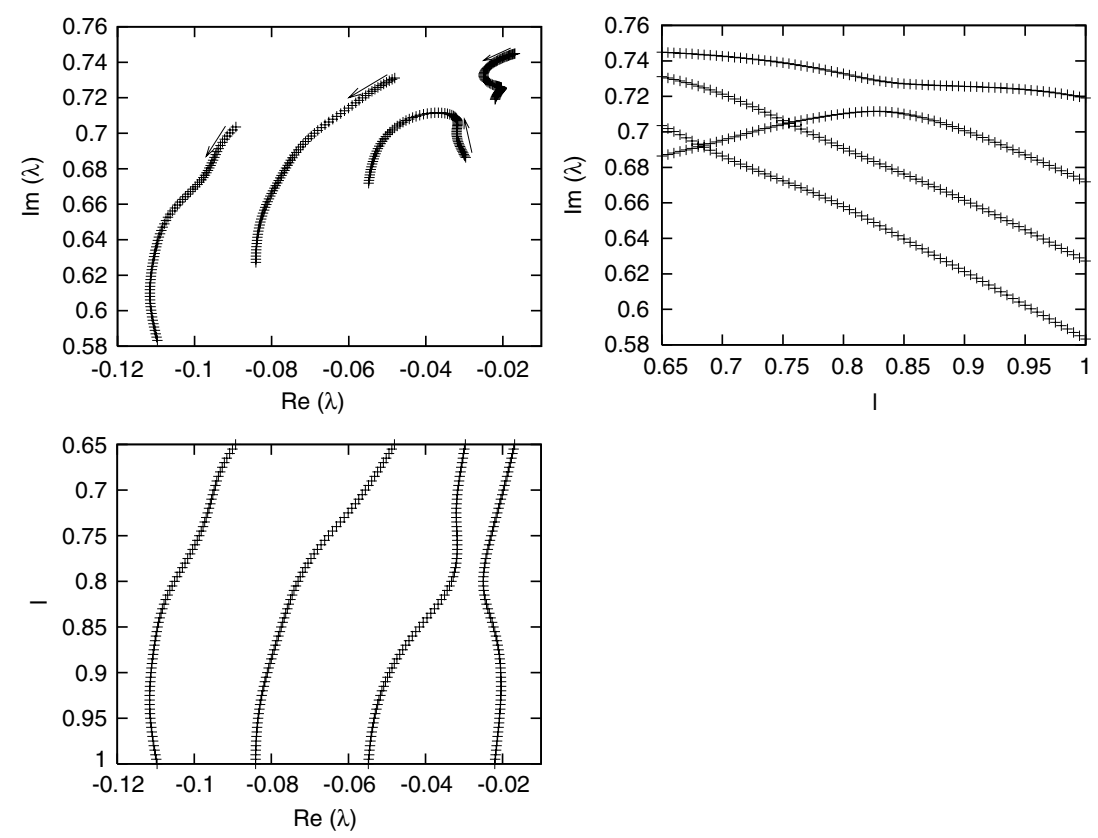

Figure 5. Top left: the resistive spectrum as $l$ increases. The horizontal axis is $\operatorname{Re}(\lambda)$, while the vertical axis is $\operatorname{Im}(\lambda)$. The figures are for $l$ from 0.65 increasing to $1 . \log \eta=-4, k_{z}=0.25$ and $k_{y}=1.3$. The arrows indicate the starting point of the frequencies (i.e. the spectrum for $l=0.65$ ) and their initial direction as $l$ increases. Top right: $\operatorname{Im}(\lambda)$ versus $l$ for the same parameters as the top left. Bottom left: $l$ versus $\operatorname{Re}(\lambda)$ for the same parameters as the top left.

and continues on the path of the original quasi-mode. This avoided crossing is confirmed by the analogous features visible in the bottom left panel $(\operatorname{Re}(\lambda)$ versus $l$-plot) in figure 5 . As such, the interaction could be thought of as an exchange of quasi-mode and Alfvénic properties between the two oscillation modes as $l$ increases and the inhomogeneity spreads over the whole plasma.

This avoided crossing is not as clearly visible for every initial equilibrium configuration. For example, the interaction depicted in figure 6 does not show a clear avoided crossing behaviour. The equilibrium parameters are almost exactly the same as in figure 5, except for a different value of $k_{y}$. In the top right panel, the two interacting modes do not seem to influence the curve of the other mode significantly. In the bottom left panel, however, the curves of the resistive Alfvén and quasi-mode are initially parallel, but suddenly these parallel curves are split in two distinct levels when $l \approx 0.78$. This is exactly the point where the paths of the Alfvén and quasi-mode intersect in the top right panel. Although there is no clear evidence of an avoided crossing, the quasi-mode is really converted into an Alfvénic mode.

Because the damping of the resistive Alfvén modes is dependent on the number of nodes in the radial direction (Kerner et al 1986), the modes closest to the imaginary axis have one node. The next one on this spectral branch has two nodes, etc. Since this strict ordering is disturbed by the interaction with the original quasi-mode, we might also find indications of interactions when looking at the number of nodes in the eigenfunctions during the interaction.

Figure 7 shows the eigenfunctions of the top three modes plotted in figure 5 before, during and after the interaction. This figure clearly shows that a node is added to the eigenfunction of the quasi-mode during the process and that the number of nodes of the Alfvén mode right 

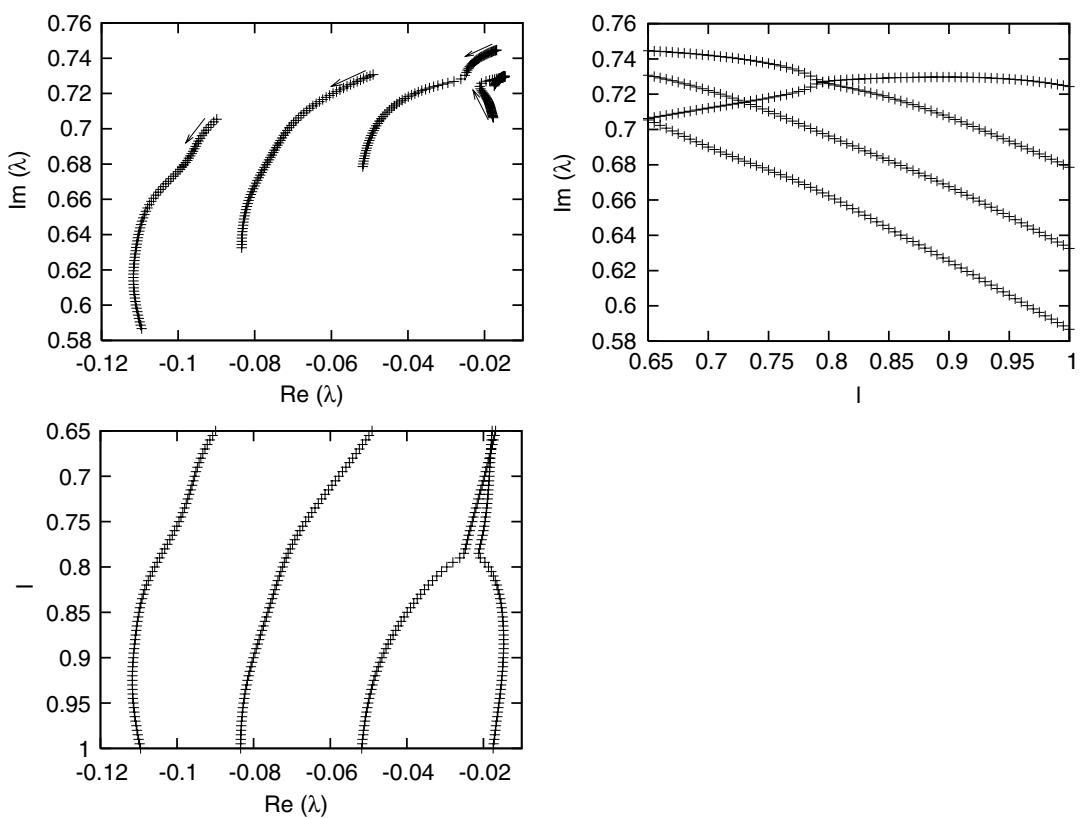

Figure 6. Top left: the resistive spectrum as $l$ increases. The horizontal axis is $\operatorname{Re}(\lambda)$, while the vertical axis is $\operatorname{Im}(\lambda)$. The figures are for $l$ from 0.65 increasing to $1 . \log \eta=-4, k_{z}=0.25$ and $k_{y}=1.4$. The arrows indicate the starting point of the frequencies (i.e. the spectrum for $l=0.65$ ) and their initial direction as $l$ increases. Top right: $\operatorname{Im}(\lambda)$ versus $l$ for the same parameters as the top left. Bottom left: $l$ versus $\operatorname{Re}(\lambda)$ for the same parameters as the top left.

of the quasi-mode is decreased to zero. The number of nodes of the Alfvén mode left of the quasi-mode remains unaltered. It seems that in this case, when the quasi-mode moves into the discrete resistive spectrum, the Alfvén modes right of the quasi-mode lose a node. For other equilibrium parameters, it is less clear which Alfvén modes lose/gain a node and whether the strict ordering on the resistive branches still exists after the interaction.

To stress the exchange of wave properties as the equilibrium parameters change, figure 8 plots the resistive spectrum when, respectively, changing (1) $l$ from 0.63 to 1 (red + ), (2) $\eta$ from $10^{-3.2}$ to $10^{-3.5}$ (green $\times$ ) and (3) $l$ from 1 to 0.63 (blue $*$ ). It is observed that during step (1) the quasi-mode (red arrow with III) initially moves to the left and is converted to a resistive Alfvén mode. It positions itself among the other modes on the branches of the resistive Alfvén spectrum. When $\eta$ is decreased (step 2), all modes move up the branch of the resistive Alfvén spectrum (as depicted in figure 3). In the third step, $l$ is decreased to its original value. During this step, a resistive Alfvén mode exits from the resistive Alfvén branch and becomes the quasi-mode (blue arrow with II). The new quasi-mode was originally a resistive Alfvén mode, and the original quasi-mode is now a resistive Alfvén mode. It is thus clear that an exchange of wave properties occurred while changing the equilibrium parameters.

This picture stresses the fact that the interaction stands for an exchange of wave properties. As $l$ increases, the original quasi-mode passes its properties on to resistive mode II. When $\eta$ is decreased, the eigenfunctions and physics of the system remain unchanged. When $l$ is decreased, resistive mode II emerges as the quasi-mode out of the resistive spectrum. This proves that the original quasi-mode (III) has passed its wave properties on to eigenmode II when $l$ was changed. 

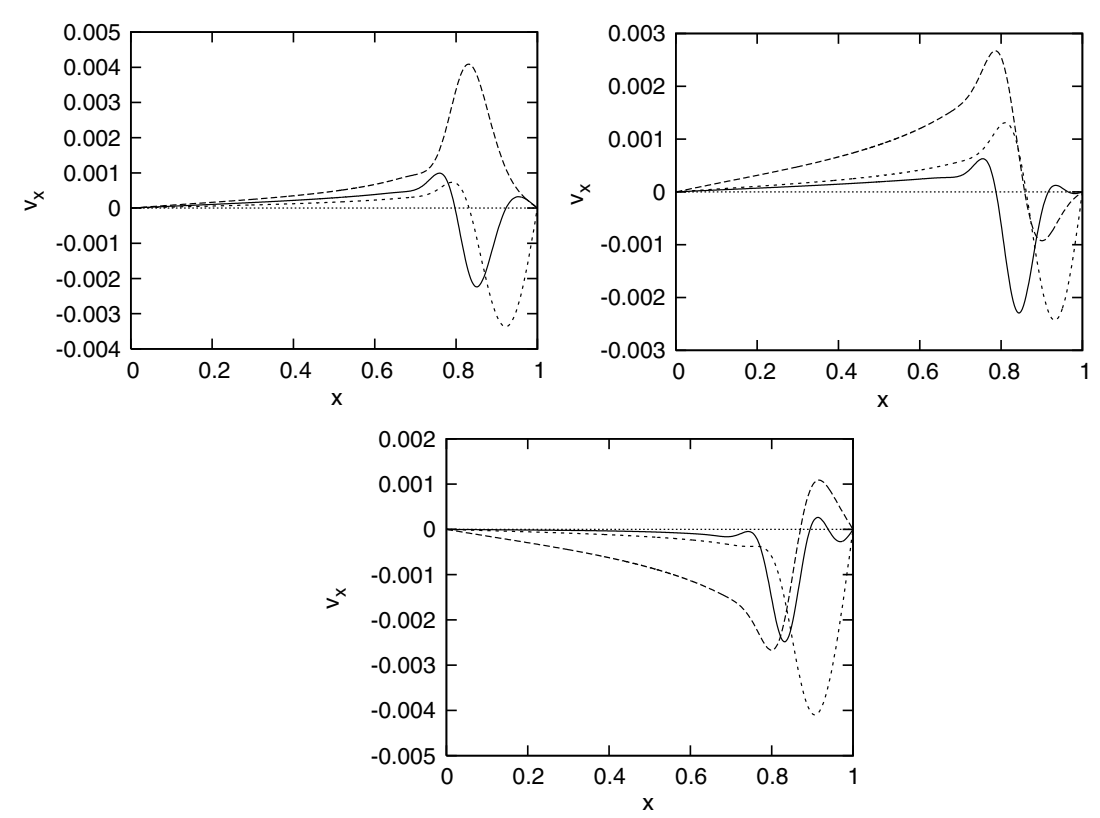

Figure 7. $v_{x}$ versus $x$ for $\log \eta=-4, k_{z}=0.25$ and $k_{y}=1.3$. The top left panel is for $l=0.74$, top right panel for $l=0.77$ and the bottom panel for $l=0.8$. The continuous line is the Alfvén mode left of the quasi-mode, the long-dashed line shows the original quasi-mode and the short dashes are for the Alfvén mode right of the quasi-mode.

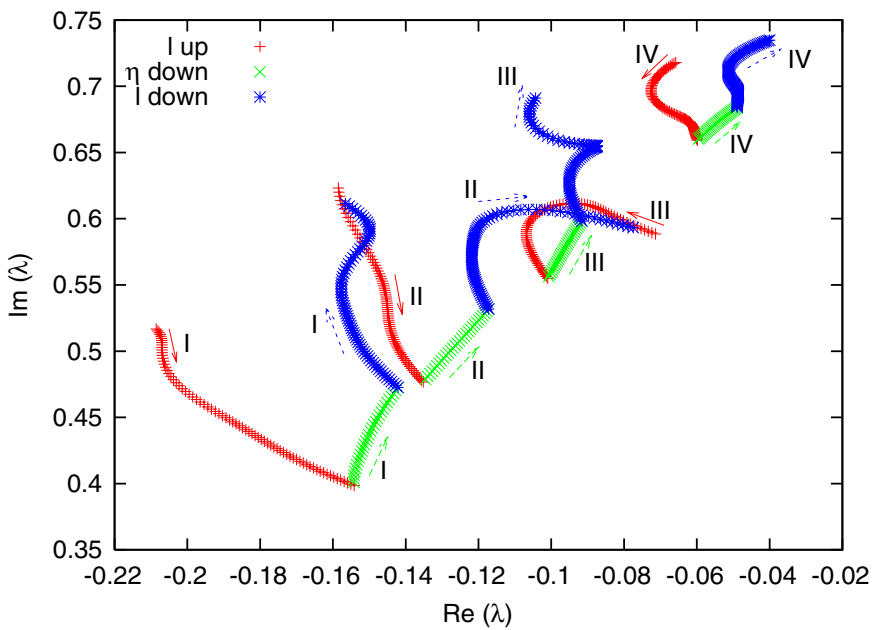

Figure 8. The resistive Alfvén spectrum for $k_{z}=0.25$ and $k_{y}=1 . l$ is changed from 0.63 to 1 (red +), followed by a decrease of $\eta$ from $10^{-3.2}$ to $10^{-3.5}$ (green $\times$ ) and a decrease of $l$ from 1 to $0.63\left(\right.$ blue $\left._{*}\right)$.

\section{Consequences}

Our calculations may be of importance in the field of coronal loop seismology. We could take our plasma slab as a crude model for a coronal loop and assume that the coronal loop is oscillating in the fundamental kink quasi-mode. We could try to determine the density contrast 


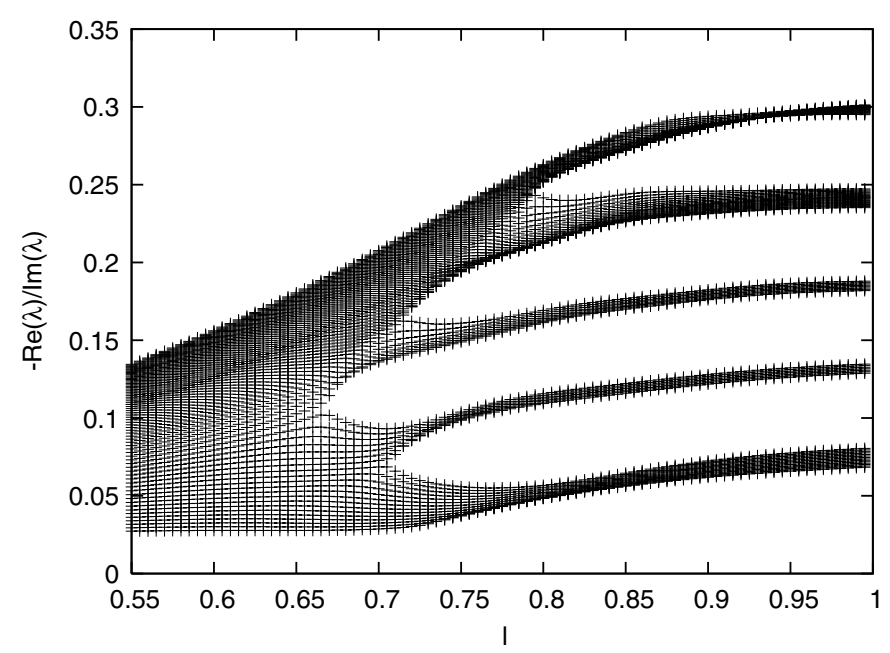

Figure 9. $-\operatorname{Re}(\lambda) / \operatorname{Im}(\lambda)$ versus $l$ for $\log \eta=-4, k_{y}=1$. In this plot $\zeta$ increases from 5 to 12 in steps of 0.1 .

and $l$ in a given coronal loop with an observed damping rate. To do this, we plot $-\operatorname{Re}(\lambda) / \operatorname{Im}(\lambda)$ (proportional to the damping rate, see Aschwanden et al 2003) versus $l$ for different values of the density contrast $\zeta$. Such a figure is displayed in figure 9. By drawing a horizontal line at the observed damping rate, we can restrict $l$ and $\zeta$ to a narrower interval. Thus, we find information about the immediate surroundings of the considered coronal loop.

In the abovementioned figure $-\operatorname{Re}(\lambda) / \operatorname{Im}(\lambda)$ is plotted versus $l$ for $\zeta$ going from 5 to 12 in steps of 0.1 . Several gaps are clearly visible. In these gaps, $-\operatorname{Re}(\lambda) / \operatorname{Im}(\lambda)$ can never fit the observed damping rate. These prominent gaps are caused by an Alfvén mode interacting with the studied quasi-mode. Because of the interactions, the quasi-mode is forced to choose a side of the Alfvén mode, either left or right (respectively, below and above the gap). Different gaps belong to interactions with different resistive Alfvén modes.

Because Alfvén modes are very sensitive to the resistivity $\eta$, the gaps are highly mobile when $\eta$ changes. Therefore, if $\eta$ is not constant in the corona, the gaps may still be covered when taking different values for $\eta$. For a fixed value of $\eta$, however, forbidden strips in the complex frequency plane are formed. It is impossible for a quasi-mode frequency to enter these forbidden strips for any value of $l$ and $\zeta$.

It is clear that these forbidden strips result in a quantization of the damping rate $-\operatorname{Re}(\lambda) / \operatorname{Im}(\lambda)$. For a given $l(\geqslant 0.8)$, only strictly separated values for the damping rate can be attained, thus resulting in an effective quantization of the damping rate. Because of the presence of the discrete resistive modes, a discretization and quantization of the quasi-mode frequency is obtained.

Gaps similar to those in figure 9 are also found in more sophisticated one- and twodimensional cylindrical models for a coronal loop (Arregui et al 2005).

\section{Conclusions}

In this paper, we have shown that the quasi-mode frequency cannot cross the branches of the resistive Alfvén spectrum when the inhomogeneity of the system is increased. Instead a strong interaction takes place. During this interaction, avoided crossings are observed and the Alfvén 
modes between the quasi-mode and the imaginary axis lose a node in their eigenfunction. After this interaction, the quasi-mode seems to be converted into a discrete resistive eigenmode and no differences between the original quasi-mode and the resistive Alfvén modes are observed.

In our calculations, it seems that, when the medium is inhomogeneous enough, the quasimode disappears from the resistive spectrum. Therefore, it seems that the one-to-one relation between the resistive and the ideal quasi-mode is lost. Will it be necessary to change our picture of the quasi-mode and the resistive spectrum? Do our calculations mean that coronal loops which have a radially non-uniform density profile cannot oscillate as a kink quasi-mode?

We were unable to explain the observed features and could not determine the cause of these interactions. A lot of open questions thus remain.

We did point out, however, that our calculations may be of particular importance for coronal loop seismology. Since these interactions are the cause of forbidden strips in the complex frequency plane, certain physical parameters of coronal loops are unable to reproduce the observed oscillations and damping rate. This means that, from a coronal seismologists point of view, given an observed set of frequency and damping rate, certain sets of physical parameters for the coronal loop models can be excluded.

\section{References}

Andries J, Arregui I and Goossens M 2005 Astrophys. J. 624 L57-60

Andries J, Goossens M, Hollweg J V, Arregui I and Van Doorsselaere T 2005 Astron. Astrophys. 430 1109-18

Arregui I, Van Doorsselaere T, Andries J, Goossens M and Kimpe D 2005 Astron. Astrophys. 441 361-70

Aschwanden M J, Fletcher L, Schrijver C J and Alexander D 1999 Astrophys. J. 520 880-94

Aschwanden M J, Nightingale R W, Andries J, Goossens M and Van Doorsselaere T 2003 Astrophys. J. 598 $1375-86$

Balet B, Appert K and Vaclavik J 1982 Plasma Phys. 241005

Dewar R L and Davies B 1984 J. Plasma Phys. 32 443-61

Goedbloed J P and Poedts S 2004 Principles of Magnetohydrodynamics (Cambridge : Cambridge University Press)

Kerner W, Lerbinger K, Gruber R and Tsunematsu T 1985 Comput. Phys. Commun. 36 225-40

Kerner W, Lerbinger K and Riedel K 1986 Phys. Fluids 29 2975-87

Nakariakov V M and Ofman L 2001 Astron. Astrophys. 372 L53-6

Nakariakov V M, Ofman L, DeLuca E E, Roberts B and Davila J M 1999 Science $285862-4$

Poedts S and Kerner W 1991 Phys. Rev. Lett. 66 2871-4

Riedel K S 1986 Phys. Fluids 29 1093-104

Ruderman M S and Roberts B 2002 Astrophys. J. 577 475-86

Ryu C M and Grimm R C 1984 J. Plasma Phys. 32 207-37

Schrijver C J et al 1999 Sol. Phys. 187 261-302

Van Doorsselaere T, Andries J, Poedts S and Goossens M 2004 Astrophys. J. 606 1223-32

Van Doorsselaere T, Debosscher A, Andries J and Poedts S 2004 Astron. Astrophys. 424 1065-74 